\section{The brain on microarrays}

\author{
Microarrays for the Neurosciences: An Essential Guide \\ Edited by Daniel H. Geschwind and Jeffrey P. Gregg \\ MIT Press, \$55.00/£36.95 (cloth), ISBN 0-262-07229-7, 2002
}

\section{Reviewed by Jeanne F. Loring ${ }^{1}$ and P. Scott Eastman ${ }^{2}$}

${ }^{1}$ Arcos BioScience, 3550 General Atomics Court, San Diego, California, USA. ${ }^{2}$ Incyte Genomics, 3160

Porter Drive, Palo Alto, California, USA.

Mention microarrays to a biologist and you are sure to evoke a response. More often than not, the response will be a shudder or a curse. Biologists are suspicious of microarray data, and reasonably so; many published microarray studies are wrong, and many more simply provide uninteresting lists of genes. All technologies go through stages. Like microarrays, Southern blotting, DNA sequencing and transgenics all started as techniques exclusive to a few laboratories. As any new technique starts to 'catch on', it goes through a stage in which its valuable applications are rapidly outstripped by its misuses. Microarray technology is at this stage now. Many researchers want to use microarrays but are not exactly sure why or how, and, as a result, they make mistakes. The book Microarrays for the Neurosciences is a timely publication that provides concrete examples from laboratories that have used (and perhaps misused) array technology. The

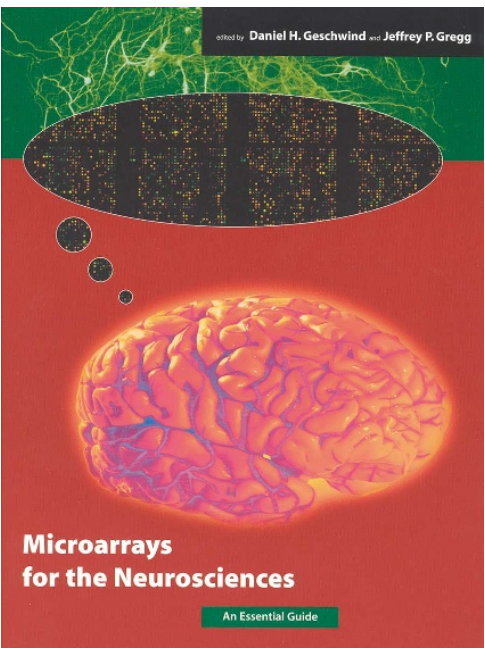

And yes, several chapters do include lists of genes. But it would be hard to find a better overview of array-based applications for complex biological systems in one reference.

There is something for everyone. The chapter by Awad and colleagues, for example, offers a clear description of how to use Affymetrix's oligonucleotide arrays, with a refreshing emphasis on experimental design over analytical methods. Experienced researchers will be disappointed, however, that there is no description of any of the flexible methods that allow empirical testing of oligonucleotides on arrays, such as spotting and in situ synthesis on slides (as used by Agilent, for example). The chapter by Shah and colleagues presents a nice discussion of image analysis mechanisms and information management without getting bogged down in mathematical details. Pickett's chapter provides an excellent dispurpose of the book is neatly summarized in the last sentence of the introduction: it urges neuroscientists to "have a chip on the bench, rather than on their shoulder." (It should be noted that the book discusses both microarrays and larger-format filter arrays; although it is not formally defined, the term "microarray" is generally reserved for high density, slide-sized arrays.)

This is not the perfect book for the microarray-wary, nor will it satisfy the old hand. Although the book was based on a short course offered at the Society for Neuroscience meeting in 1998, it lacks the coherence of a set of lessons. In spite of the editors' intention, the chapters are an uneven assortment of down-to-earth methods and more theoretical discourse. the requirements for setting up a lab of one's own, and the chapters by Becker and by Chiang show that large-format filter arrays are a practical way to do gene expression analysis without making a big investment. The introduction to statistical analysis by Nadon is a perfect reference for biologists who would rather experiment than calculate. Van Deerlin's summary of methods for amplifying RNA from fixed tissue is particularly useful to neuroscientists, as so much information about central nervous system pathologies is locked in archived histological sections.

There are some shortcomings to the book. Probably the most important one is the book's failure to dispel some of the persistent misperceptions about microarrays, such as that microarrays are intrinsically quantitative; that a twofold difference in gene expression is automatically significant; and that it doesn't matter how much DNA is spotted on arrays when they are used for competitive hybridizations.

We like to learn from others' mistakes, as well as our own, and would have preferred to see more discussion of the pitfalls of microarray technology. For example, what do you do when different expression methods (such as quantitative PCR and microarrays) give different results? What about the 'garbage in, garbage out' problem? Quality control is the bugaboo of commercial suppliers, and researchers want to know which quality control methods can be used for either homemade or purchased arrays. Other important issues, such as how to extract information at low signal levels, are not discussed. And although signalto-noise calculations are covered, there is no discussion of which value should be used for background subtraction: global signal, mismatched sequences, element background?

In sum, this book will be useful because it takes a practical approach to introducing a new technology, and remembers that the brain of the biologist is still the most important tool for neuroscience research. There will be time for one, or perhaps two, new editions of this book and we look forward to reading them. But we also agree with the editors' implication that in a few years, microarrays will be just another routine technique, and some other technology will be in vogue for use, and misuse, by researchers who don't want to miss out on the latest trend. 